\title{
Packet Scheduling Distributed Online Weighted Algorithm on Inter-satellite Links
}

\author{
Ma Xiao ${ }^{1}$ \\ ${ }^{1}$ School of Computer and Telecommunication Engineering, University of Science and \\ Technology Beijing
}

\begin{abstract}
Satellite communication system includes inter-satellite links (ISL) for two terminals which are located in different coverage of two satellites. Proper mechanism of packet scheduling is needed to maximum the link efficiency after the ISL created. In this paper, a packet scheduling distributed online weighted algorithm on ISLs was advanced. This algorithm is fully taken into account to the limited loads and the real-time data transfer requirements of satellite communication system.
\end{abstract}

Keywords: satellite; ISL; distributed online algorithm; weighted

\section{Introduction}

The satellite communication system includes mainly about GEO (geostationary satellite), MEO and LEO (medium earth orbit and Low earth orbit) satellites [1].

The GEO satellite is not suitable for real-time communication because of its long propagation delay. The LEO satellite propagation delay is $6 \sim 70 \mathrm{~ms}$ [2], which is fit for real-time operations.

In the Communication satellite system, ISLs are needed if two terminals are located within the coverage of the different satellites after an end-to-end call is established by two end users. On the path, a traffic scheduling mechanism should be proposed to maximize efficient use of the link by the packets selection and to avoid minor transmission excessive occupying the ISLs.

\section{Related Works}

As to the scheduling problem of communication network, the attempt of solving method includes scheduling algorithm based on frame structure [3][4]. In this method, the fixed-length time slots are included in every frame. The packets are stored in buffer, and then be transmitted in the next frame [5][6]. But this method is mainly targeted at terrestrial networks, not consider the characteristics of the satellite network with ISLs.

The process of solving scheduling problem is to arrange different serving in the same path reasonable, and to defuse the conflict arising from different services seizing the path. But there is no traffic scheduling algorithm for satellite communications network with ISLs.

\section{The algorithm researching}

\subsection{Model}

The channel multiplexing method is that each ISL is divided in $\mathrm{K}$ channels. 
When the packets are transmitted, all the channels are working simultaneously. The satellite nodes data transceiver uses frame structure. Each frame contains the same number of fixed-length packets. When a path enters a high-load state, the scheduling is needed for the transmitting packet.

\subsection{Service Classification and Defini- tion}

We proposed an objective function based on integer programming to describe the process of packets selecting in a satellite network, and limit the packet selection range by the constraint.

After the path established, let $S_{i}(i=1,2, \ldots, m)$ denote the in-path satellite nodes, and the bandwidth is band, $($ band $=1,2, \ldots, k)$, the maximum value is equal to the length of $\mathrm{k}$ packets.

Denote $T,(T=1,2, \ldots, t)$ as the system running time. $t=t+1$ when all the nodes complete a round of packets receiving from ground terminals, selecting and transmitting and forwarding process. The packet in the satellite buffer waiting for transmitting over the ISLs is $(i=1,2, \ldots, m, j=1,2, \ldots, n)_{i}$, and the source node of the packet is $S r c_{i j}$, the destination node is Dest $_{i j}$. The weight of the packet in buffer is

$$
w_{i j}, i=1,2, \ldots, m-1, j=1,2, \ldots, n_{i} .
$$

In order to arrange the ISL capacity to various types of services, we distinguish the service type of the packet by $w_{i j}$.

The packet weight is defined in (1):

$$
w_{i j}=\text { type }_{i j} \cdot\left(1+e^{-\frac{1}{2} h}\right)
$$

Where parameter type $e_{i j}$ is the service types:

$$
\text { type }_{i j}= \begin{cases}10, & \text { VOD } \\ 5, & \text { VoIP } \\ 1, & \text { Data services }\end{cases}
$$

We let $h$ as hops which a packet is transmitted from source node to the destination node, and $1+e^{-\frac{1}{2} h}$ is defined as hop_factor. When the hops of transmitted packet are increasing, more hops the transmitted packets are, lower the hop_factor is.

$\delta_{i j q}$ is to reflect that the packet whether on the path at node q. The value of $\delta_{i j q}$ indicates the relative position of packet $(i, j)$ and destination node Dest $_{i j}$, shown in (3):

$$
\begin{gathered}
\delta_{i j q}: \delta_{i j q}=\left\{\begin{array}{l}
0, \delta_{i j q} \leq q \\
1, \delta_{i j q}>q
\end{array}\right. \\
\delta_{i j q}=0 \text { indicates that node } \mathrm{q} \text { is after }
\end{gathered}
$$

Dest $_{i j}$ so that the transmission is end before packet $(i, j)$ reaching node $\mathrm{q}$ and the channel at node $\mathrm{q}$ is no longer occupied. Whereas $\delta_{i j q}=1$ indicates that node $\mathrm{q}$ is in front of Dest ${ }_{i j}$. The channel is still occupied at node $\mathrm{q}$ because of node $\mathrm{q}$ is a relay node on the routing path.

\subsection{The maximum weighted distrib- uted scheduling online algorithm}

We suppose that the number of satellites on the path is $\mathrm{m}$ and the last one only receiving packets but not sending or forwarding. Therefore, the first satellites involves in the data transmission and forwarding. Each satellite can store $n_{i}$ packets in the buffer. In each transmission period, the largest number of satellite node sent on ISL is K. 
To maximizing the throughput of the path, we aim to find top value of $w_{i j}$ under above condition.

$x_{i j}$ describes the packet $(i, j)$ selection result. Let $x_{i j}=1$ if the $\operatorname{packet}(i, j)$ is selected to transmit on the path. By contrast, $x_{i j}=0$ if it isn't selected.

$$
\begin{aligned}
& x_{i j}=\left\{\begin{array}{l}
1, \text { selected } \\
0, \text { unselected }
\end{array}\right. \\
& \left(i=1,2, \ldots, m-1, j=1,2, \ldots, n_{i}\right)
\end{aligned}
$$

S.T.

$$
\begin{aligned}
& \sum_{i=1}^{q-1} \sum_{j=1}^{n_{i}} \delta_{i j q} x_{i j}+\sum_{j=1}^{n_{q}} \delta_{i j q} \leq k, \\
& q=1,2, \ldots, m-1 \\
& x_{i j}=0 \text { or } x_{i j}=1, \\
& \left(i=1,2, \ldots, m-1, j=1,2, \ldots, n_{i}\right)
\end{aligned}
$$

The (5) shows that the number of packets transmitted by each node cannot exceed the ISL capacity $\mathrm{K}$ in current time.

We propose a distributed online algorithm to solve the problem above .

The greedy algorithm is applied in the packets selecting process. Each satellite is traversing all the $w_{i j}$ of packets in its buffer in the same time, and then first $l$ packets with $\max w_{i j}$ are selected to transmitting if the idle link can send $l$ packets from current node $S_{i}$ to the next node $S_{n+i}$.

We aim to maximize the sum of packets weight transmitted by the node. Let $t=0$ after the system initialized, $t=t+1$ after each time period.

\section{Performance Evaluation}

\subsection{Simulation Model}

Our packets scheduling algorithm and the FIFO(first-in/first-out) scheduling al- gorithm are separately run under the same constellation model in order to compare two algorithms performance of weight and the number of total transmitted packets.

We use Iridium-like constellation. Each satellite maintains four ISLs with its neighboring. Average packet length is $1.5 \mathrm{~kb}$ with $4 \mathrm{Mbit} / \mathrm{s}$ bandwidth. Channel multiplexing is used in ISLs, and the numbers of channels are 2, 4, 6 and 8 .

The result is taken after 1000 time periods each experiment and for 10 times.

\subsection{Simulation Results}

1) The total weight of transmitted packets: Fig. 1 shows the performance in different channel numbers.

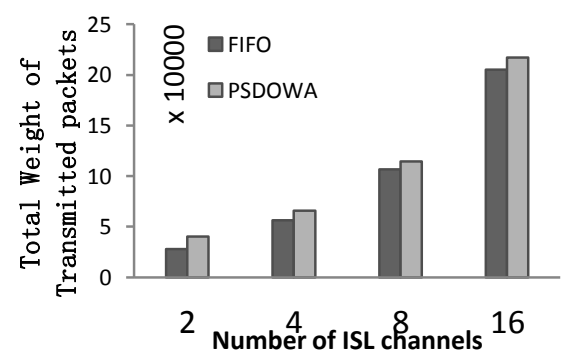

Fig.1: The weight of total transmitted packets

The total weight of transmitted packets is calculated in (7).

$$
W=\sum_{i=1}^{m-1} \sum_{j=1}^{n_{i}} w_{i j} \mathrm{x}_{i j}
$$

The average improved ratio of the total weight of the transmitted packets between PSDOWA and FIFO is calculated in (8) and the result shown in table 1 .

$$
\begin{aligned}
& \Delta\left(\overline{\sum_{i=1}^{m-1} \sum_{j=1}^{n_{i}} w_{i j} \mathrm{x}_{i j}}\right)_{P S D O W A} \\
& \left.-\Delta \overline{\left(\sum_{i=1}^{m-1} \sum_{j=1}^{n_{i}} w_{i j} \mathrm{x}_{i j}\right.}\right)_{F I F O}=10547
\end{aligned}
$$


Tabel.1

The average improved ratio of the total weight between PSDOWA and FIFO

\begin{tabular}{|c|c|c|c|c|}
\hline $\begin{array}{c}\text { Channel } \\
\text { Number }\end{array}$ & 2 & 4 & 6 & 8 \\
\hline $\begin{array}{c}\text { Improved Ra- } \\
\text { tio(\%) }\end{array}$ & 45.11 & 17.15 & 7.61 & 5.80 \\
\hline
\end{tabular}

When the number of channels is increasing, the improved ratio of total weight is decreasing but PSDOWA is still take advantage.

It shows that the total weight is more than FIFO in PSDOWA. In the case of the high occupancy rate of ISLs, PSDOWA can ensure the transmission of important communication services.

2) The total numbers of transmitted packets: Fig. 2 shows the changes of total transmitted numbers of packets in 2,4,6 and 8 channels in ISLs. Fig. 4 shows that in PSDOWA, the total numbers rises in 5\% more that FIFO schedule mechanism. The results show that more packets are transmitted in PSDOWA thus the utilization of the link has been improved.

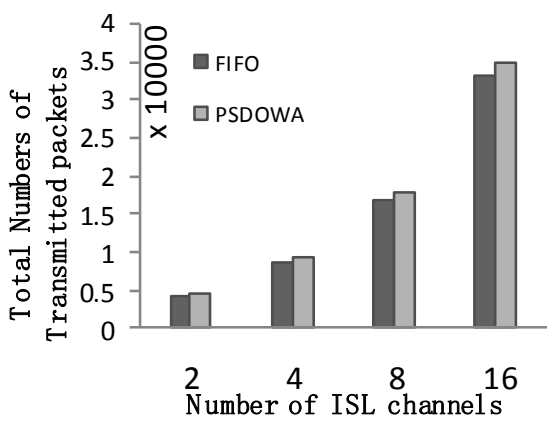

Fig.2 The number of total transmitted packets

\section{Conclusion}

In this paper, we proposed a packet scheduling distributed online weighted algorithm on inter-satellite links (PSDOWA). The algorithm gives the appropriate weight to each packet into the satellite buffer with their type of services and number of hops. Fully considering the limitation of satellite communications system load, we designed distributed computing and online algorithms to improve the scheduling mechanism. The simulation results shows that the weight of transmitted packets improved 5.8\%$45.11 \%$ and the number of packets improved 5\% in different bandwidth of ISLs under PSDOWA.

\section{References}

[1] John Farserotu, Ramjee Prasad, "A Survey of Future Broadband Multimedia Satellite System-Issues and Trends", IEEE Communication Magazine, June 2000, Vo1.38, No.6: 128-133

[2] Chotikapong Y, Cruickshank H, and Sun Z L, "Evaluation of TCP and Internet traffic via low Earth orbit satellites," IEEE Personal Communications, 2001, 8(3):28-34

[3] T. Lee and C. Lam, "Path Switching-A Quasi-Static Routing Scheme for Large Scale ATM Packet Switches, "IEEE J. Selected Areas in Comm., vol.15, no.5, pp.914-924, June 1997

[4] S. Li and N. Ansari, "Input-Queued Switching with QoS Guarantees," Proc.IEEE INFOCOM '99, pp. 1152-1159, 1999

[5] M. Andrews and L. Zhang, "Achieving Stability in Networks ofInput-Queued Switches, " Proc. INFOCOM '01, pp.1673-1679, 2000

[6] M.A. Marsan , E. Leonardi, M. Mellia, and F. Neri, "On the Throughput Achievable by Isolated and Interconnected Input-Queued Switches under Multiclass Traffic," Proc. INFOCOM '02, pp.16051614, 2002 